\title{
El discurso político y su reflejo en los medios: la credibilidad en tiempos de crisis (2008-2011)
}

\author{
Laura CANDÓN GAUTIER \\ Universidad de Málaga \\ laura_cg22@hotmail.com \\ Carmen Delia MÁRQuez RuIZ \\ Universidad de Málaga \\ carmenlau4@hotmail.com
}

\section{Resumen:}

La crisis económica de los últimos años ha derivado en una crisis de credibilidad tanto de la política como de los medios de comunicación. Esta cuestión se ha convertido en el punto neurálgico del debate sobre el papel que ambos actores juegan en una sociedad democrática.

Palabras clave: Discurso; política; credibilidad; agenda setting

\section{Political discourse and its reflection in the media: credibility in times of crisis (2008-2011)}

\begin{abstract}
:
The economic crisis of recent years had led to a crisis in the credibility of politics as well as of the media. This has become the central issue in the debate as to the roles both parties play in a democratic society.
\end{abstract}

Key Words: Discourse; credibility; agenda setting

Referencia normalizada:

Candón Gautier, L. y Márquez Ruiz, C. D. (2014): El discurso político y su reflejo en los medios: la credibilidad en tiempos de crisis (2008-2011). Historia y Comunicación Social. Vol. 19. Núm. Especial Marzo. Págs. 129-137.

Sumario: 1. Introducción. 2. La agenda política y la agenda mediática. 3. Audiencias y credibilidad. 4. Metodología. 5. Resultados. 6. Conclusiones. 7. Referencias bibliográficas

\section{Introducción}

Para tratar la cuestión de la credibilidad del discurso político y mediático, hemos de partir del contexto en el que los dos actores encuentran su punto de acción común y desgranar el papel que juega cada uno. Los estudios sobre comunicación política que se han desarrollado en los últimos años, han puesto su foco de atención en el 
solapamiento entre los mensajes políticos y el discurso mediático, puesto que, con el asentamiento de las sociedades democráticas y, sobre todo, ante la aparición de la televisión, la idea generalizada en el entorno académico es que nos encontramos en un sistema "mediocrático", esto es, en una democracia que tiene lugar en los medios de comunicación. (Swanson, 1995a: 4)

\section{La agenda política y la agenda mediática}

Con el objetivo manifiesto de conseguir el apoyo necesario de la ciudadanía y obtener la legitimación necesaria para sostenerse, las instituciones políticas se han amoldado a la dinámica mediática, adaptando sus estrategias y su discurso para influir en su agenda:

A medida que las noticias televisivas se establecen como la primera fuente de información para el público, aumenta la rivalidad entre los medios de información y los funcionarios del gobierno por controlar el orden de los acontecimientos y por determinar cuales serán considerados como asuntos de importancia y cómo serán presentados (Swanson, 1995b: 3-4).

Por tanto, en esta investigación tenemos en cuenta la estrategia informativa de los medios, estudiada desde la perspectiva de la agenda setting. Autores como Lippmann (2003) o McCombs (2006) han destacado la gran capacidad de los medios para influir en la percepción ciudadana, estableciendo, en un primer nivel los temas que han de tenerse en cuenta y, en un segundo, la manera en que éstos deben enfocarse, construyendo así una realidad específica. La mayoría de los estudios que han partido de este punto, han investigado el tratamiento de la agenda compuesta por temas de interés público y han establecido relaciones basadas en la capacidad de los medios de comunicación para influir en los procesos de formación de la opinión pública.

Sin embargo, esta estrategia no siempre consigue el éxito esperado. Para que éste se produzca, los medios de comunicación deben encontrar una correspondencia entre los temas que esgrimen y los temas relevantes para la audiencia. En este sentido, la teoría de agenda setting ofrece dos elementos que ayudan a la comprensión de esta circunstancia: la clasificación de los temas en cercanos (obstrusive) y lejanos (unobstrusive). En el primer caso, la audiencia tiene una relación directa con el elemento mediatizado, por lo que tiende a fiarse más de su experiencia, mientras que, si se expone a una representación mediática de temas lejanos, suelen asumirla por no contar con otras fuentes que las descarten. 


\section{Audiencias y credibilidad}

En una situación marcada por una fuerte crisis económica, que en palabras de Timoteo Álvarez (2012) ha puesto de manifiesto la incapacidad de la socialdemocracia y del neoliberalismo para dar respuesta a ella, se ha producido de forma paralela una fuerte crisis de confianza que también incluye a los medios de comunicación.

Por tanto, y como señala Díaz Nosty (2005: 7) "la credibilidad se ha convertido en la cuestión central del debate sobre los medios y, con ello, el cuestionamiento general sobre el papel que éstos juegan en una sociedad democrática"

Partiendo de estos supuestos teóricos, el objetivo de esta investigación radica en el análisis de la agenda política para, a su vez, hacer lo propio con la agenda mediática entre los años 2009 y 2011 en España. Pretendemos observar si los medios de comunicación utilizan una agenda propia en una situación de crisis económica, o si por el contrario, asumen el discurso de las instituciones políticas para, posteriormente, comparar los resultados con las preocupaciones de los ciudadanos. Asumimos que los medios de comunicación ejercieron un rol crítico con respecto a la política en estos años, sin embargo, partimos de la idea de que no existió una correspondencia entre la agenda de los medios y los intereses de la audiencia, algo que ha desembocado en una falta de credibilidad tanto de la política como de los medios.

\section{Metodología}

Para ello, hemos basado la investigación en la aplicación del método cuantitativo a través de un análisis de contenido a los discursos de José Luis Rodríguez Zapatero y Mariano Rajoy durante los debates sobre el estado de la nación dados entre 2009 y 2011. Tras identificar los temas tratados por ambos, se han comparado con los resultados de un análisis de la misma naturaleza de las informaciones dadas sobre política nacional en los periódicos El País y El Mundo durante la semana posterior al debate. La elección de ambas publicaciones viene determinada por el objetivo de cubrir las dos líneas ideológicas predominantes en los medios de comunicación en España.

\section{Resultados}

5.1. 2009

\subsubsection{Agenda política}

En la agenda política del año 2009, los principales temas abordados fueron en lo referente al Partido Socilista: la educación, la normalización del sector inmobiliario, 
las medidas sociales y ETA. En lo que respecta al Partido Popular, el desempleo, la crisis, el déficit público y la falta de previsión de Zapatero fueron sus temas prioritarios.

\subsubsection{Agenda mediática}

La agenda de los medios refleja los temas abordados por el PSOE (gráfica 1) y por el PP (gráfica 2) tal y como se detalla a continuación.

\subsubsection{Temas propios del PSOE:}

\section{Grafica 1}

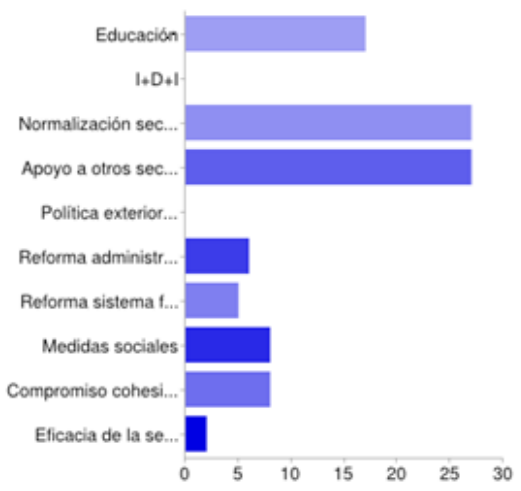

Educación $17 \%$

$1+D+10 \%$

Normalización sector inmobiliario $27 \%$

Apoyo a otros sectores $27 \%$

Política exterior (crisis global) $0 \%$

Reforma Administración de Justicia $6 \%$

Reforma sistema de financiacióna autonómica $5 \%$

Medidas sociales $8 \%$

Compromiso cohesión social e igualdad $8 \%$

Eficacia de la seguridad/ETA $2 \%$

\subsubsection{Temas propios del PP}

\section{Gráfica 2}

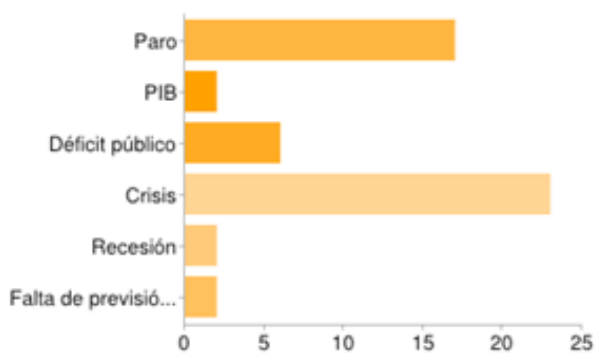

Paro $33 \%$

PIB 4\%

Déficit público $12 \%$

Crisis $44 \%$

Recesión $4 \%$

Falta de previsión de Zapatero $4 \%$ 


\subsubsection{Intereses de los ciudadanos}

El paro es el tema que más preocupa a la sociedad española (79,3\%), así como los problemas de índole económica (48,7\%). La inmigración aparece con un 16,3\%, a continuación el problema del terrorismo y ETA con un $15,5 \%$, la inseguridad ciudadana $(11,4 \%)$ y para finalizar se muestra con el $10,1 \%$ los políticos en general, los partidos políticos y la política.

\subsection{0}

\subsubsection{Agenda política}

Los temas proclamados por el presidente del Partido Popular, Mariano Rajoy son aquellos relacionados con la situación de crisis económica que atraviesa España, entre los que destacan: la austeridad en el gasto público, medidas para fomentar el empleo y críticas a Zapatero en lo que refiere a la gestión de la crisis económica. Por otro lado el presidente del Gobierno proclama en el debate del Estado de la Nación del año 2010 los siguientes: necesidad de cooperación, diálogo y trabajo continuo, economía y empleo, el Estatuto de Cataluña ETA y la seguridad.

\subsubsection{Agenda mediática}

La agenda mediática del diario El País y El Mundo, reflejan los principales temas proclamados por el Partido Socialista (gráfica 3) y el Partido Popular (gráfica 4).

\subsubsection{Temas del PSOE}

\section{Gráfica 3}

Temas del PSOE

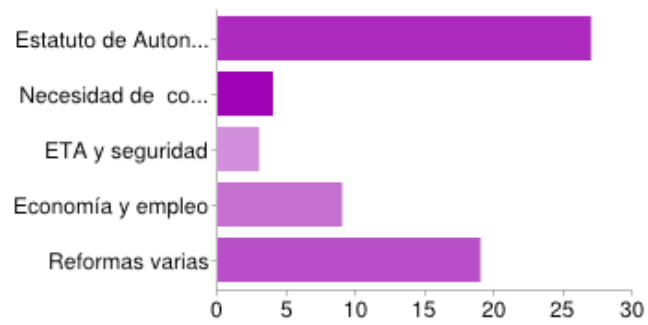

Estatuto de Autonomía $44 \%$

Necesidad de cooperación, diálogo y

trabajo coninuo $6 \%$

ETA y seguridad $5 \%$

Economía y empleo $15 \%$

Reformas varias $31 \%$ 
5.2.4 Temas PP 2010

\section{Gráfica 4}
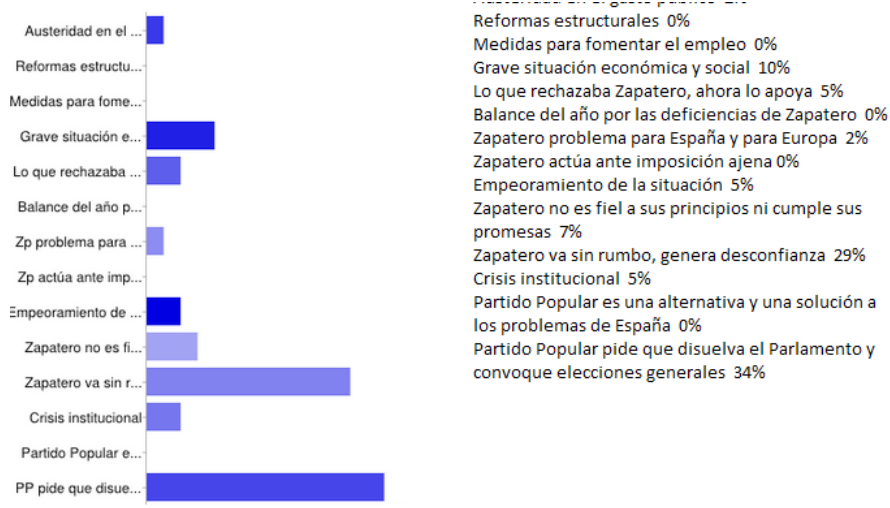

Partido Popular es una alternativa y una solución a los problemas de España $0 \%$

Partido Popular pide que disuelva el Parlamento y convoque elecciones generales $34 \%$

\subsubsection{Interés de los ciudadanos}

En el año 2010, paro es el principal problema para los españoles (78\%) seguidos con un 51,3\% de los problemas de índole económica. Los políticos en general, los partidos políticos y la política han pasado a ser el tercer problema de los españoles en el año 2010.

\subsection{1}

\subsubsection{Agenda política}

Entre los temas abordados por el Partido Socialista destacan la crisis económica, el marco laboral, la austeridad, la crisis de credibilidad y las reformas para cambiar el modelo productivo. Por otro lado el Partido Popular trató temas como: la crisis económica, el paro, la deuda pública, la prima de riesgo, educación, Zapatero como causante de la crisis y su petición de convocar elecciones anticipadas, existiendo por tanto una relación con los temas que los diarios han dado mayor relevancia (gráfica 5 у 6$)$. 


\subsubsection{Agenda mediática}

\subsubsection{Temas PSOE 2011}

\section{Gráfica 5}

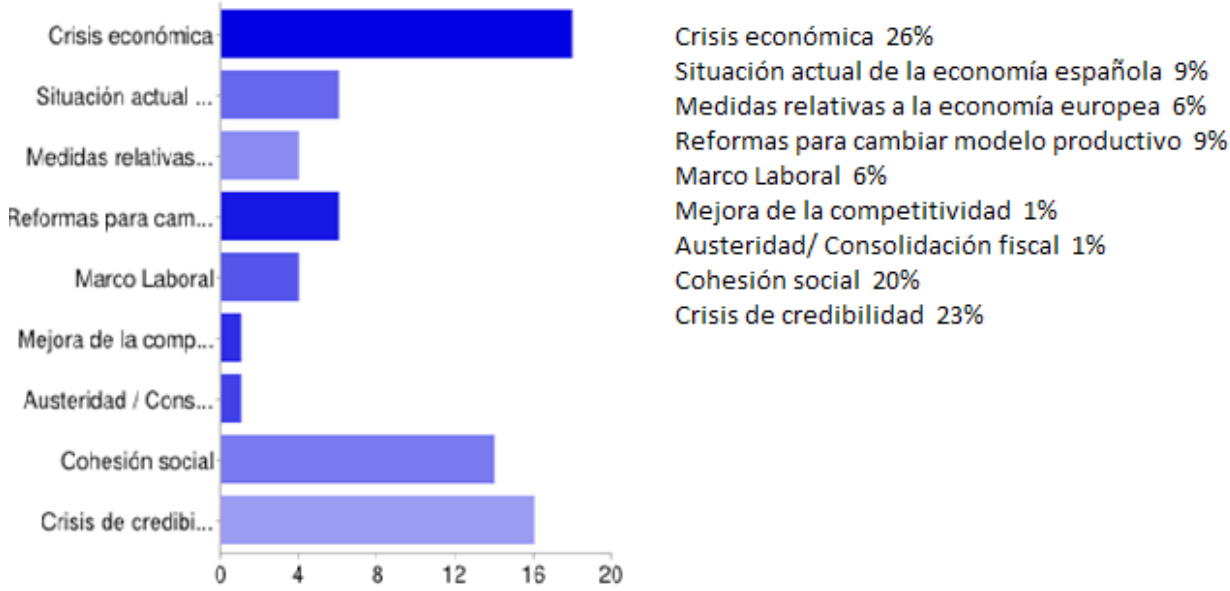

5.3.4 Temas PP 2011

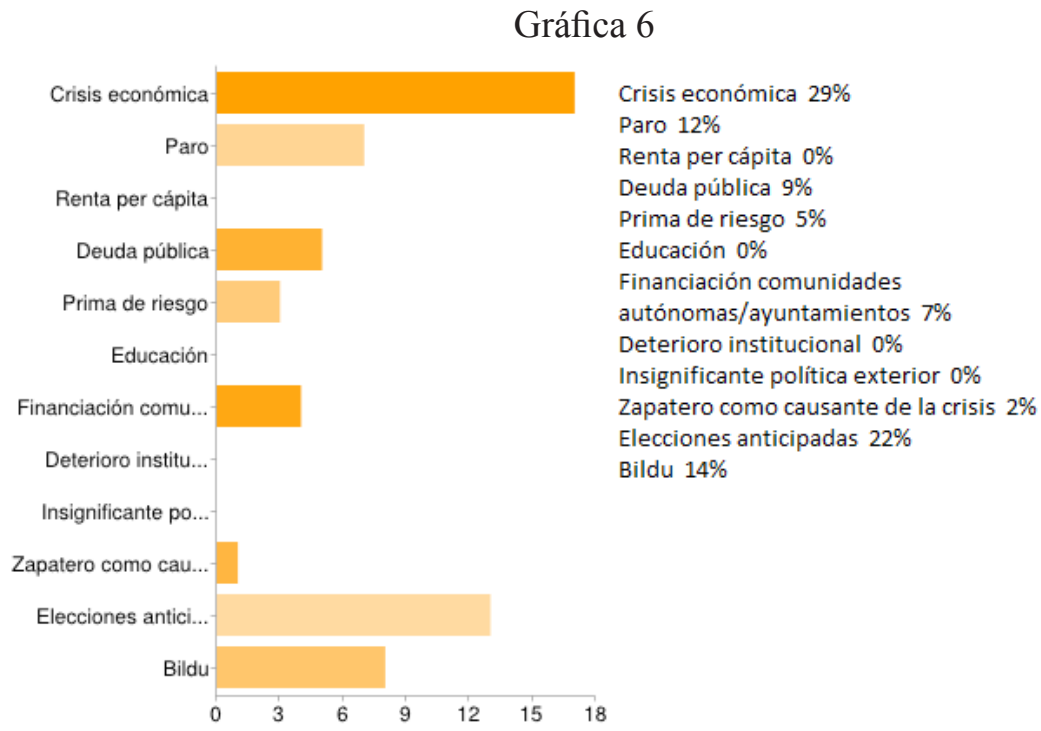




\subsubsection{Interés de los ciudadanos}

En el año 2011, el paro es el problema que más afecta a los españoles con un $81,3 \%$ de los españoles, seguido de los problemas de índole económica (49,6\%). Un año más, vuelve a subir el porcentaje relativo a los políticos en general, los partidos políticos y la política, con un $24 \%$.

\section{Conclusiones}

Los diarios analizados se hacen eco de los principales temas abordados por los líderes políticos en los debates sobre el Estado de la Nación, reflejando por tanto intereses políticos. En el diario El Mundo tienen mayor protagonismo las noticias cuyo eje central está dedicado a la crítica al Gobierno y a su despedida, complementando la información con las propuestas de la oposición. El diario El País se caracteriza por incluir en la mayoría de las noticias analizadas, información neutral del Gobierno, publicando declaraciones a favor y en contra del mismo, al igual que ocurre con la oposición, a pesar de que se muestre una tendencia favorable a las prácticas llevadas a cabo por el Partido Socialista.

Los diarios analizados muestran las preocupaciones más relevantes de la sociedad española, que están relacionadas con el paro, la crisis, y los problemas económicos, publicados con un enfoque político y reflejando las medidas y propuestas que los dirigentes han expuesto en el debate del Estado de la Nación, dando más importancia a los intereses de los partidos que a los de los ciudadanos.

El País y El Mundo no reflejan en sus publicaciones temas que preocupan a los ciudadanos como la violencia machista y la inmigración. La sección "España" da mayor prioridad a temas políticos que tienen como protagonistas tanto a socialistas como populares, sin reflejar temas de actualidad nacional que preocupa a los españoles.

\section{Bibliografía}

DÍAZ NOSTY, B. (2005): El déficit mediático. Donde España no converge con Europa. Bosch. Barcelona.

KRIPPENDORF, K. (1997): Metodología de análisis de contenido. Teoría y práctica. Paidós. Barcelona.

LIPMANN, W. (2003): La opinión pública. Cuadernos de Langre. Madrid.

McCOMBS, M. (2006) Establecimiento de la agenda: El impacto de los medios en la opinión pública y el conocimiento. Paidós. Barcelona. 
SWANSON, David L. (1995): El campo de la Comunicación Política. La democracia centrada en los medios, en MUÑOZ-ALONSO, A. \& ROSPIR, J.I.: Comunicación política. Universitas S.A. Madrid.

RODRÍGUEZ DÍAZ, R. (2004): Teoría de la Agenda-Setting. Aplicación a la enseñanza universitaria. Disponible en: http:/www.obets.ua.es/obets/libros/AgendaSetting.pdf. [22-08 2013].

TIMOTEO ÁLVAREZ, J. (2012): Manejo de la comunicación organizacional. Espacios, herramientas y tendencias en gestión de negocios. Díaz de Santos. España.

URIARTE, E. (2001): La crisis de la imagen de la política y de los políticos y la responsabilidad de los medios de comunicación. Disponible en: http://dialnet. unirioja.es/servlet/articulo? codigo $=27646 \&$ orden $=0 \&$ info $=$ link. $[8-08-2013]$

\section{Las autoras}

Laura Candón Gautier: Licenciada en Periodismo por la Universidad Carlos III de Madrid y actual estudiante del Máster en Investigación en Comunicación Periodística de la Universidad de Málaga. Mi experiencia profesional se relaciona con los medios de comunicación regionales y locales, como redactora y locutora en Cadena Ser Radio Jerez en el año 2010 y redactora en Diario de Cádiz (Grupo Joly) entre 2011 y 2012. Poseo nivel de inglés alto, acreditado con la P2 (Prueba de inglés para licenciados de la Universidad Carlos III de Madrid) y nivel A2 en francés, acreditado por la misma Universidad.

Carmen Delia Márquez Ruiz, licenciada en periodismo en la Universidad de Málaga y alumna del Máster de Investigación en Comunicación Periodística de la mencionada universidad. Mi experiencia profesional se encuentra relacionada con los medios de comunicación locales, entre los que destacan: RONDA TV (12 meses), RONDA SEMANAL (GRUPO INFORMACIÓN) (12 meses), CHARRY TV (6 meses), RADIO RONDA (4 meses) y LA VOZ DE RONDA. Tengo un conocimiento alto de Inglés hablado y escrito, y el nivel A1 en Alemán por la Escuela Oficial de Idiomas. 